\title{
Experimental determination of the transversal and longitudinal fibre bundle permeability
}

\section{Journal Article}

Author(s):

Schell, J.S.U.; Siegrist, M.; Ermanni, P.

Publication date:

2007

Permanent link:

https://doi.org/10.3929/ethz-b-000009148

Rights / license:

In Copyright - Non-Commercial Use Permitted

Originally published in:

Applied composite materials 14(2), https://doi.org/10.1007/s10443-007-9035-1 


\title{
Experimental Determination of the Transversal and Longitudinal Fibre Bundle Permeability
}

\author{
J. S. U. Schell • M. Siegrist • P. Ermanni
}

Received: 9 January 2007 / Accepted: 18 June 2007 /

Published online: 4 August 2007

(C) Springer Science + Business Media B.V. 2007

\begin{abstract}
In the resin transfer moulding process, fabrics are very often used as reinforcement. These fabrics consist of fibre bundles. In this context, both the permeability of the macroscopic fabric and the permeability of the fibre bundle are the key parameters to accurately predict the impregnation of the fabrics, the impregnation time and resulting void content. The fibre bundle permeability can be either predicted theoretically or experimentally. Whereas the theoretical determination lacks on accuracy as the realistic packing of the fibres in the bundles is not integrated in the models. In this work, we present an experimental setup to measure the longitudinal and transversal permeability of fibre bundles. The results are compared to model predictions.
\end{abstract}

Keywords Fibre bundle $\cdot$ Permeability measurement

\section{Introduction}

Resin transfer moulding process is a resin injection process. Layers of a reinforcement are laid in a mould, in which a resin is injected to impregnate the fibres and the shape forming occurs. The reinforcement is very often a woven, stitched or braided fabric. A fabric, made up of fibre bundles which themselves consist of thousands of fibres, is a dual scale medium. Consequently, the impregnation of a fabric is a dual-scale flow [1-3]. Depending on the resin velocity and the relation of the fibre bundle permeability to the inter-bundle permeability, the resin flows faster inside the bundles or in the inter-bundle channels.

J. S. U. Schell · M. Siegrist · P. Ermanni ( $\otimes)$

ETH Zurich, Centre of Structure Technologies, Leonhardstr. 27, CH-8092 Zurich, Switzerland e-mail: permanni@ethz.ch

J. S. U. Schell

e-mail: jschell@ethz.ch

URL: www.structures.ethz.ch 
To describe and predict the impregnation time and process of the fabrics, permeabilities on both scales, bundles and inter-bundle region, need to be known.

These permeabilities are required to simulate the impregnation of a fabric predicting the necessary injection time and the resulting void content [4].

The permeability of the inter-bundle region refers to the saturated permeability of the macroscopic fabric. The characterisation of the in-plane permeability of a fabric has been a matter of research in Han et al. [5], Verheus and Peeters [6] and Luthy et al. [7]. Today procedures are well established. Measurements of the fibre bundles permeability are rare. Some experimental determinations of longitudinal permeability of fibre bundles are described in literature [8-10]. In Ranganathan [11] a model of elliptical solid cylinders is investigated. The cylinders represent in one case the fibres in the bundles to predict the bundle permeability $K_{b}$ and in a second case the cylinders are porous with the permeability $K_{b}$ to determine the permeability of the macroscopic reinforcement.

In this work, an experimental method is presented to determine the transversal and longitudinal saturated bundle permeability, which takes care on the fibre arrangement during the sample preparation. This paper is structured in 4 parts: In Section 2 the theory of flow through fibre bundles modelled with Darcy's law including a review of the literature concerning the fibre bundle permeability is presented. The experimental approach and device are explained in Section 3. The experimental results are presented and discussed in Section 4. The conclusions of this work are presented in Section 5.

\section{Theory}

The flow through the bundles is described as the flow through a porous material. This is a typical problem in hydrogeology. Several researchers discussed this flow problem [12-15] that can be described by Darcy's law:

$$
\mathbf{v}=-\frac{\mathbf{K}}{\eta} \nabla \mathbf{p}
$$

where $v$ is the Darcy-velocity, the flow of the resin penetrating the fibres, $K$ the permeability of the preform, $\eta$ the viscosity of the resin and $\nabla p$ the pressure gradient [16]. Darcy's law is only valid for a Newtonian fluid and for the saturated flow.

The permeability $K$ is a second order tensor describing the hydraulic conductivity of a porous medium. It has two different components: $K_{L}$ for the permeability longitudinal to the fibre axis and $K_{T}$ transversal to the axis, see Fig. 1:

$$
\mathbf{K}=\left[\begin{array}{ccc}
K_{L} & 0 & 0 \\
0 & K_{T} & 0 \\
0 & 0 & K_{T}
\end{array}\right]
$$

There are several equations which describe the permeability such as the well known Kozeny-Carman equation developed for description of granular isotropic porous media. In this equation, the permeability of a granular medium is a function of the porosity $\phi$ and of the radius $r$ of the spherical granules:

$$
K=\frac{r^{2}}{4 k} \frac{\phi^{3}}{(1-\phi)^{2}}
$$


Fig. 1 Fibre bundle with the directions of the longitudinal permeability $K_{L}$ and the transversal permeability $K_{T}$

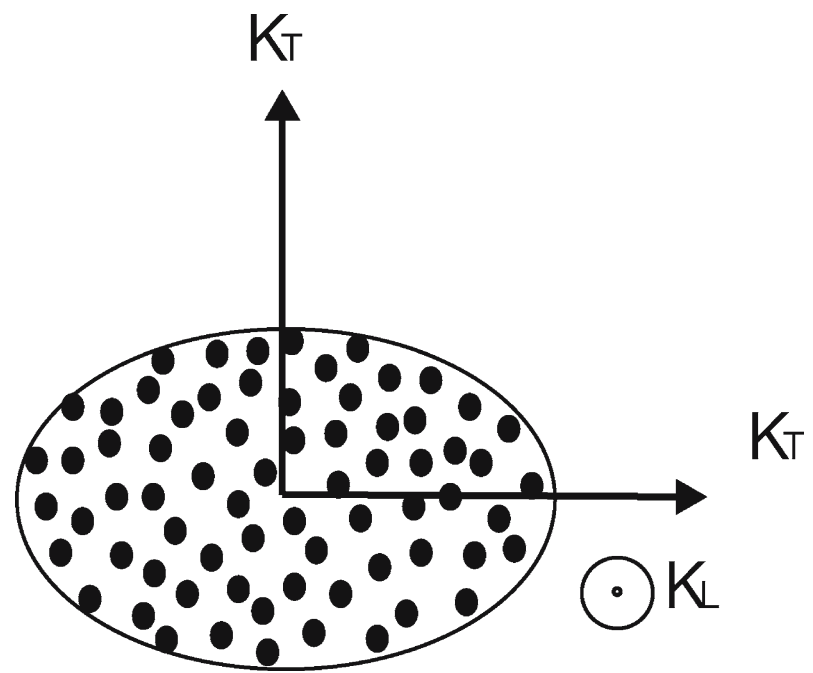

where $k$ is the empirical Kozeny constant. The permeability increases with increasing porosity. $[17,18]$ In the case of a fibre bed, $r$ is the average radius of the fibres.

The Carman-Kozeny equation is proposed as an approach to approximate the longitudinal and transversal fibre tow permeability by Gutowski [10]. In experiments the differences between the theory and reality are identified. The review in [19] gives the same estimation of the Carman-Kozeny equation: it is very simply to use, only the Kozeny constant has to be determined. But this parameter has to join the theory with reality. An overview of the fibre bundle permeability models in textile processes is given in [20]. The problems of the Kozeny-Carman equation are presented.

Skartsis et al. [21] reviewed the literature of fluid flow through porous media. They concentrated especially on the Kozeny constant $k$. This constant is not suited to describe the flow through aligned fibre beds as it does not take into account bed nonuniformities like varying capillary diameters. $k$ is not constant over large porosity ranges. The authors found that for axial flow the theory predicts lower permeabilities than can be experimentally determined. In their own experiments, Skartsis et al. [22] found this dependence on the fibre bed and additionally a dependence on the test fluid and on the pressure-drop.

A similar review is given by Astrom et al. [23]. They compared experimentally and theoretically determined values of the Kozeny constant $k$. The authors show the discrepancies between the $k$ determined with an ideal fibre bed model to the $k$ based on a realistic fibre bed prediction or experimental determination. Models for the determination of $k$ have to include the imperfections of real fibre beds to be consistent with experimental results.

Williams et al. [8] investigated experimentally the flow longitudinal through aligned fibres. Based on their experiments they calculated the Kozeny constant $k$. These values are smaller than the theoretical ones. The authors explain these discrepancies with the effect of packing faults. In cured cross sections, they detected such faults. Taking into account these faults it is possible to correct the Kozeny constant $k$. 


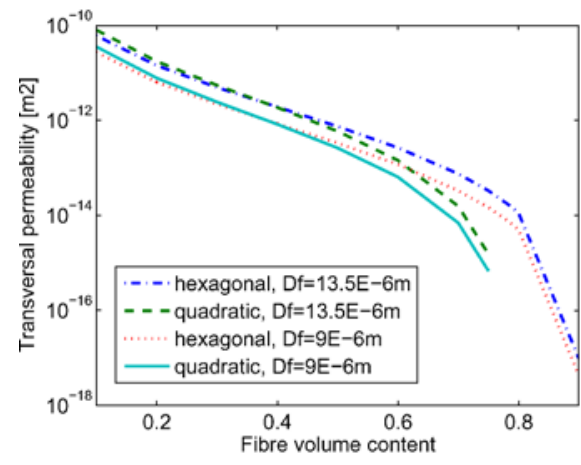

a Transversal permeability versus the fibre volume content.

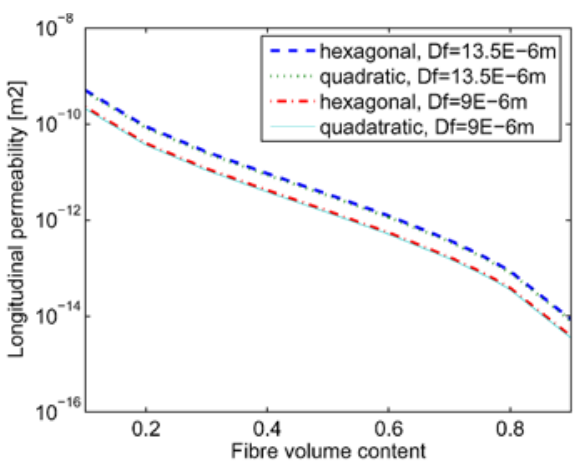

b Longitudinal permeability versus the fibre volume content.

Fig. 2 Curves of $K_{\text {trans }}$ and $K_{\text {long }}$

Gebart [24] modelled the fibre bed as regularly ordered, parallel fibres. The liquid has to flow through the gaps between the fibres. He developed an equation for the flow parallel to the fibres:

$$
K_{\text {long }}=\frac{8 R^{2}}{c} \frac{\left(1-v_{f}\right)^{3}}{v_{f}^{2}}
$$

and one for the flow transversal to the fibres:

$$
K_{\text {trans }}=C_{1}\left(\sqrt{\frac{v_{f \max }}{v_{f}}-1}\right)^{\frac{5}{2}} R^{2}
$$

where $v_{f}$ is the fibre volume fraction, $R$ the fibre radius, $v_{f m a x}$ the maximal fibre volume fraction. The two constants $c$ and $C_{1}$ depend on the fibre arrangement: hexagonal or quadratic. In the quadratic arrangement the constants are: $c=57$, $V_{\text {fmax }}=\frac{\pi}{4}, C_{1}=\frac{16}{9 \pi \sqrt{2}}$ and in the hexagonal case: $\mathrm{c}=53, V_{\text {fmax }}=\frac{\pi}{2 \sqrt{3}}, C_{1}=\frac{16}{9 \pi \sqrt{6}}$. The curves are presented for a fibre diameter of $9 \mu \mathrm{m}$ and $13.5 \mu \mathrm{m}$ in Fig. 2. The transversal permeability shows a drop at high values of $v_{f}$ and vanishes as $v_{f}$ reaches $v_{f \max }$, at which the transversal flow is blocked. The longitudinal permeability is higher than the transverse one.

Gebart compared the results of his model with a numerical approach and found good agreement. Experiments were conducted in a reinforcement of parallel fibre bundles. The flow through this unidirectional reinforcement was evaluated to get the macroscopic permeability. This macroscopic permeability is higher than the predicted bundle permeability. These studies are enlarged on the influences of

Fig. 3 Winding frame for the longitudinal measurement

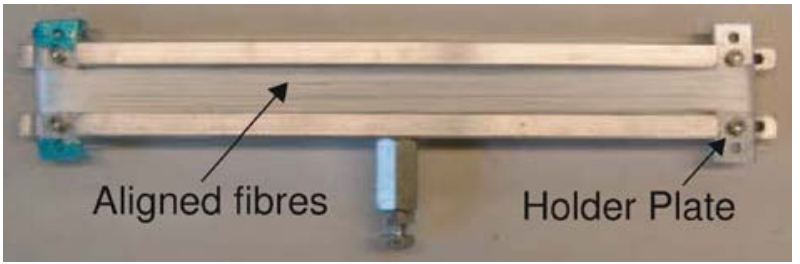


Fig. 4 The insert for the longitudinal measurement

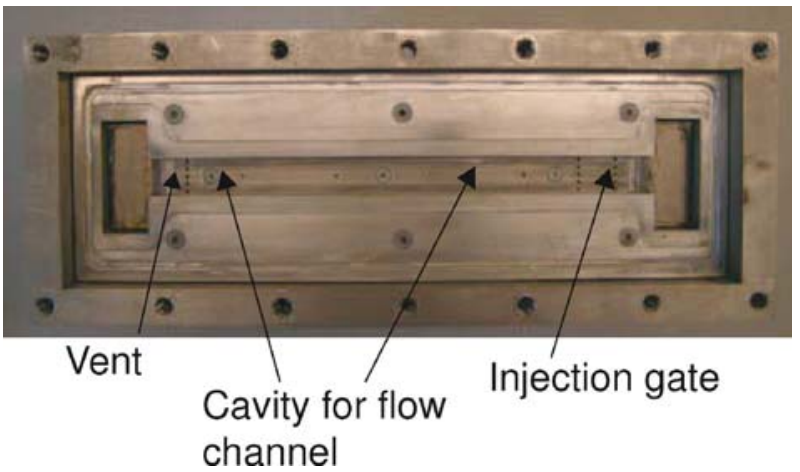

the fibre arrangement and of the fibre diameter of the fiber bundle permeability in Lundstrom and Gebart [25]. All the phenomena and their influences on the permeability are investigate separately. The single effects are non-negligible. A combination of several phenomena is missing.

Bechtold and Ye [26] investigated the influence of the fibre distribution on the transverse fibre bundle permeability. They used Morishita's quadratic method to determine the distribution of fibres in a bundle. At higher disordered fibre distribution the permeability is lower than at an ordered distribution. This disordered fibre arrangement has smaller gaps, which decreases the transverse permeability values. A similar method is presented in Um and Lee [27]. The centres of fibres were determined in cross-sections of cured fibre bundles. The positions of the fibres are taken as input in a network model to predict the bundle permeability. The network system presents the fibre bed. The predictions agree well with experimental results.

\section{Experimental}

\subsection{Principle of the Saturated Permeability Measurement}

To measure the saturated permeability, liquid is injected through completely impregnated fibres. This saturated flow is carried out at a constant injection pressure. For

Fig. 5 The insert for the transversal measurement

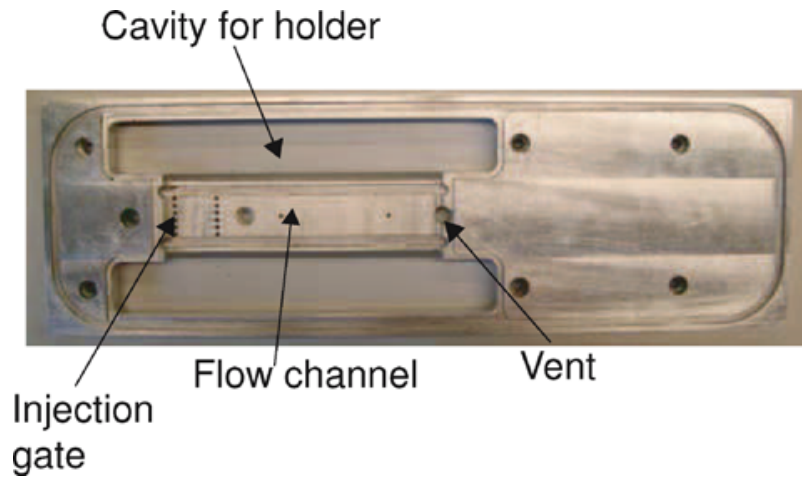

를 Springer 
Fig. 6 The insert for the longitudinal measurement with fibres

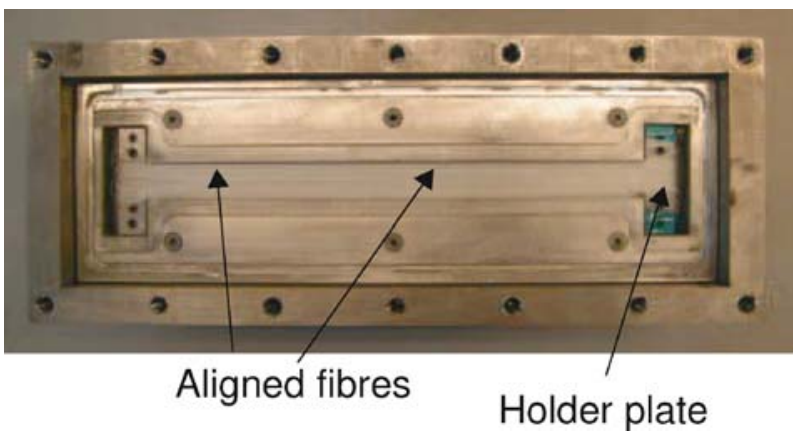

one dimensional flow Darcy-velocity is the flow rate $Q$ divided by the flow cross section $A$

$$
v=-\frac{Q}{A}
$$

For the determination of the flow rate $Q$ the mass of the fluid passing the flow channel is weighted at certain time steps. The mass per time $\dot{m}$ is calculated and divided by the density $\rho$ of the liquid to get the flow rate.

$$
Q=\frac{\dot{m}}{\rho}
$$

Permeability $K$ is calculated multiplying the Darcy-velocity with the liquid viscosity $\eta$ and the flow length $l$ divided by the pressure drop $\Delta p$

$$
K=-\frac{v \eta l}{\Delta p}
$$

\subsection{Fibre Preparation}

Roving fibre bundles are wound on a winding frame (Fig. 3). The inner diameter, the distance between the holder plates is $0.04 \mathrm{~m}$ for the transversal measurement and $0.27 \mathrm{~m}$ for the longitudinal one. The winding length is $0.13 \mathrm{~m}$ for the transversal

Fig. 7 Schematic of the insert for the transversal measurement with fibres

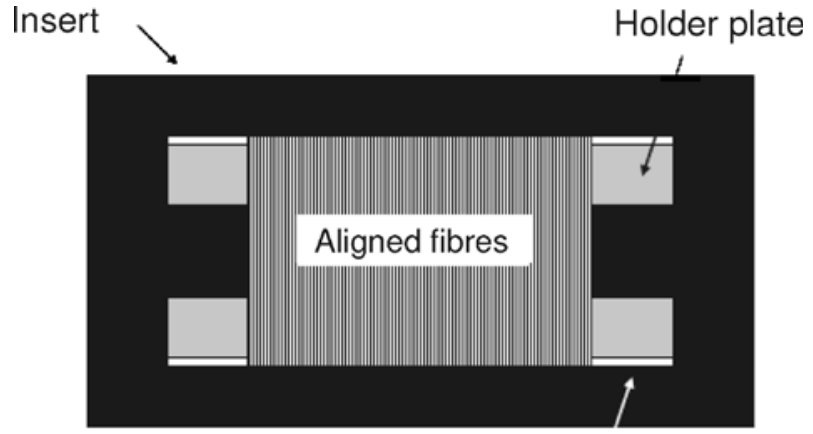

Cavity 
Fig. 8 Schematic of the insert for the transversal measurement with fibres and plasticine

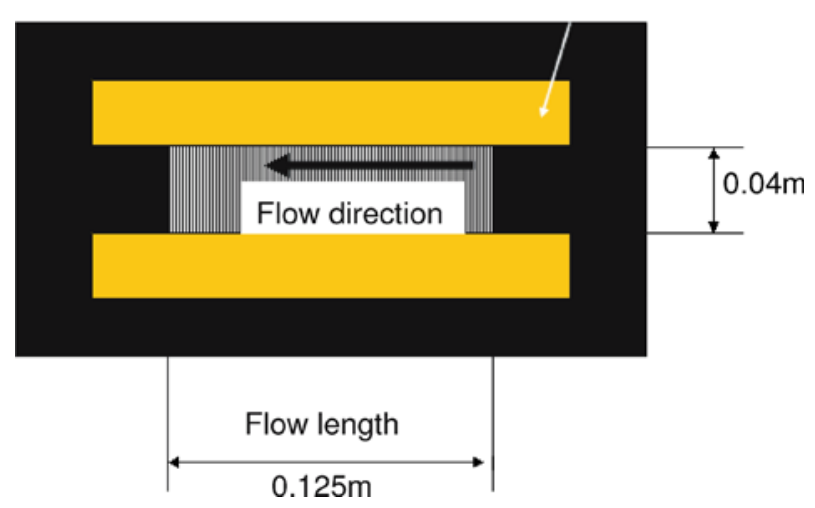

measurement and $0.02 \mathrm{~m}$ for the longitudinal one. This winding enables to fix the fibres aligned, strained and homogeneously distributed on the holder plates.

\subsection{Measurement Device}

The experimental device consists of an aluminium frame with 4 poles, a hole for liquid injection and one for the vent. Additionally, there are 4 holes to place pressure sensors and a hole for air injection. In this frame an insert for the particular measurement direction is placed. There are an insert for the longitudinal (Fig. 4) and one for the transversal (Fig. 5) permeability measurement. Each insert integrates two functions: forming the flow channel and straining the fibres.

The injection gate, the air inlet and the vent are realised with $2 \mathrm{~mm}$ wide holes. The flow length is $0.125 \mathrm{~m}$ for the transversal flow and $0.23 \mathrm{~m}$ for the longitudinal one. The cavity height is $0.009 \mathrm{~m}$, the width is $0.02 \mathrm{~m}$ for the longitudinal measurement and maximal $0.04 \mathrm{~m}$ for the transversal one.

The fibres are taken out of the winding frame and strained inside the insert. The holder plates of the fibres are fixed in cavities for ensuring the straining of the fibres (Fig. 6). In the transversal measurement case, plasticine is placed in the cavity around the holder plates and on the fibres to limit the effective flow cross section on a width of maximal $0.04 \mathrm{~m}$. Figures 7, 8 and 9 illustrate the inserts with the dimensions and flow lengths.

A polymethylmethacrylate (PMMA) plate is placed inside the flow channel, the thickness of this plate can be changed in order to vary the cavity height. A fitting flat

Fig. 9 Schematic of the insert for the longitudinal measurement with fibres

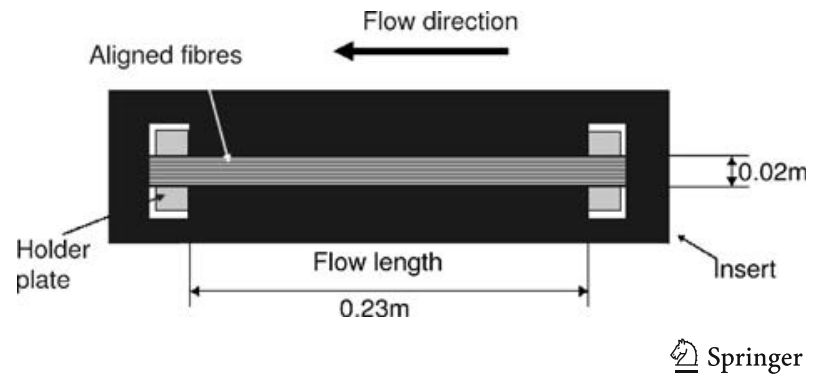


Table 1 Longitudinal permeability for different fibre volume contents $v_{f}$

\begin{tabular}{lcll}
\hline$v_{f}[\%]$ & Thread size [tex] & $K_{\text {long }}\left[m^{2}\right]$ & $\begin{array}{l}\text { Standard deviation } \\
\text { (in \%) }\end{array}$ \\
\hline 40 & 2400 & $1.7 \mathrm{E}-11$ & $5.2 \mathrm{E}-12(30 \%)$ \\
64 & 2400 & $9.6 \mathrm{E}-12$ & $3.6 \mathrm{E}-12(38 \%)$ \\
40 & 68 & $2.3 \mathrm{E}-11$ & $2.4 \mathrm{E}-12(10 \%)$ \\
58 & 68 & $1.3 \mathrm{E}-11$ & $2.9 \mathrm{E}-12(23 \%)$ \\
\hline
\end{tabular}

rubber is placed around the cavity of the flow channel. Additionally, a sealing band is put on the rubber. A $20 \mathrm{~mm}$ thick PMMA plate is clamped with a steel frame on the lower frame.

The test liquid is injected at a constant pressure using a pressure pot. The pressure at the injection gate is measured with a pressure sensor, the pressure at the vent is the atmospheric pressure. The liquid flowing out at the vent is weighted on a balance. The mass and the pressure value are recorded every second.

\subsection{Materials}

The used test liquid is a glycerin-water mixture from CHEMIA BRUGG, Switzerland. The glycerin content is $84-88 \%$ and the water content $12-15 \%$. Its density is $1.23 \mathrm{~g} / \mathrm{ml}$ and the viscosity $0.08 \mathrm{~Pa}$ s at $25^{\circ} \mathrm{C}$.

Two yarn types are used: one with a thread size of 2400 tex and a fibre diameter of $13.5 \mu \mathrm{m}$, the other has a thread size of 68 tex and a fibre diameter of $9 \mu \mathrm{m}$. These yarns have the interest to use same fibres as the fabric bundles of which the permeability is of interest. The fabric $\mathrm{A}$ is a balanced roving fabric, from Interglas, having an areal density of $600 \mathrm{~g} / \mathrm{m}^{2}$. The fabric B is a glass fibre canvas, from Interglas, having an areal density of $280 \mathrm{~g} / \mathrm{m}^{2}$. For a fibre volume fraction of $53 \%$ in fabric $\mathrm{A}$, the fibre volume fraction in the bundle is $64 \%$. This fibre volume fraction was determined using image analysis of polished specimens. A fibre volume fraction of $51 \%$ in fabric $\mathrm{B}$ results in a fibre volume fraction in the bundle of $58 \%$. The permeability measurements are carried out for these fibre volume fractions and for a $v_{f}$ of $40 \%$.

\section{Results and Discussion}

The experiments were carried out at an injection pressure of $5 \times 10^{4} \mathrm{~Pa}$, higher pressure values delivered higher permeabilities because they displaced the PMMA

Table 2 Transversal permeability for different fibre volume contents $v_{f}$

\begin{tabular}{lcll}
\hline$v_{f}[\%]$ & Thread size [tex] & $K_{\text {trans }}\left[m^{2}\right]$ & $\begin{array}{l}\text { Standard deviation } \\
(\text { in } \%)\end{array}$ \\
\hline 40 & 2400 & $3.5 \mathrm{E}-12$ & $7.2 \mathrm{E}-13(21 \%)$ \\
64 & 2400 & $4.3 \mathrm{E}-13$ & $6.7 \mathrm{E}-14(16 \%)$ \\
40 & 68 & $1.6 \mathrm{E}-11$ & $9.9 \mathrm{E}-13(6 \%)$ \\
58 & 68 & $6.9 \mathrm{E}-13$ & $7.5 \mathrm{E}-14(11 \%)$ \\
\hline
\end{tabular}


Fig. 10 Longitudinal permeability versus the fibre volume content

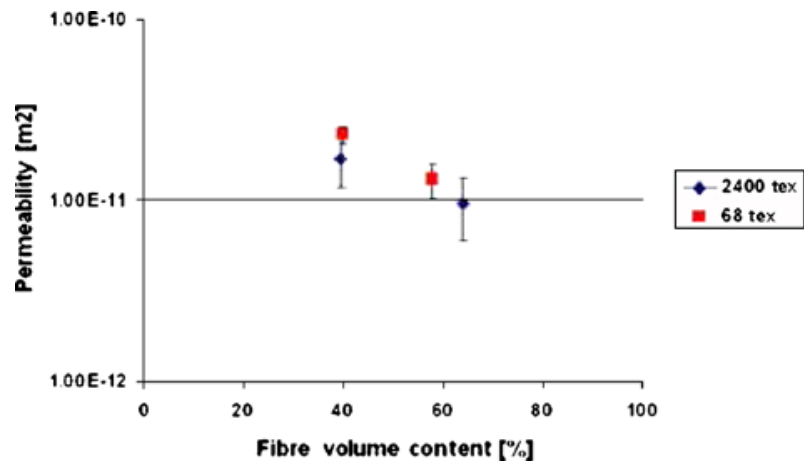

plate. This change of the permeability values is supposed to compression of the fibre bed resulting in wide flow channels. The wide channels increase the permeability.

The results for the longitudinal measurement are presented in Table 1 and for the transversal one in Table 2.

For a better illustration of the permeability values, the average values with the standard deviation are plotted on a logarithmic scale in Figs. 10 and 11.

The measured values of $K_{\text {long }}$ are superior than the values of $K_{\text {trans }}$. All values decrease with increasing fibre volume content. Both aspects are in accordance with the theory. The flow resistance is higher for the flow transversal to the fibres and at higher $v_{f}$.

The increase of $K_{\text {sat }}$ in percent with decreasing $v_{f}$ is higher for the transversal measurements. For the longitudinal measurements, the difference between $v_{f}=40 \%$ and $v_{f}=58 \%$ or $64 \%$ is small. See the logarithmic scaled graphs in Figs. 10 and 11. This can be explained with the higher possibility of flow channel creation in the longitudinal measurement. These flow channels are already created at a $v_{f}$ of $64 \%$ or $58 \%$ and reduce the longitudinal flow resistance of the fibre bed. So the flow resistance does not increase dramatically at an decrease of $v_{f}$ to $40 \%$. For the same reason, the standard deviation in percent increases with increasing $v_{f}$. At a high $v_{f}$, the occurrence of one flow channel deviates more the flow resistance than at a lower $v_{f}$. This can be seen in the standard deviation in percent in all measurements expect

Fig. 11 Transversal permeability versus the fibre volume content

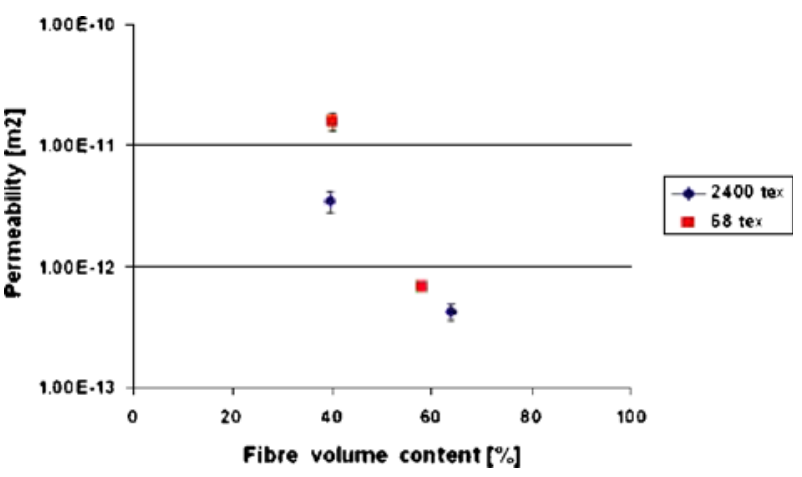


Fig. 12 Cross section of a part of a fibre bundle

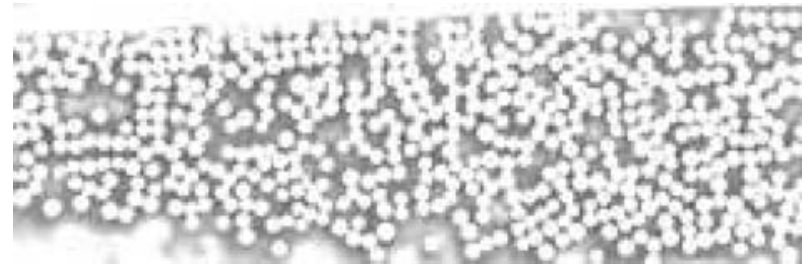

the measurement of the transversal permeability of the 2400 tex roving: the standard deviation decreases with increasing $v_{f}$.

The 2400 tex roving favours fibre bed irregularities, as these roving tends to split. This fact results in a higher disordered fibre arrangement causing higher standard deviation values in percent of the permeabilities of the 2400 tex roving compared to the values of the 68 tex roving.

The standard deviation calculated in percent is higher in the longitudinal measurements than in the transversal ones. In the longitudinal measurements, the distance between the holder plates is longer than in the transversal one. This longer distance facilitates the occurrence of realistic fibre bed irregularities, like wider flow channels, resulting in a higher standard deviation.

A comparison of all measured values with the prediction of Gebart's model [24] (see Fig. 2) shows, that the measured permeabilities are higher than the predicted ones. This coincidences with the results of Williams [8]. He found a lower flow resistance in the experiments than theoretically predicted. The real fibre bed has never the ideal fibre arrangement. Despite the perfect bundle alignment and placement in the presented measurements, the fibres are never in the ideal hexagonal or quadratic arrangement. Some differences in the packing density are inevitable.

Figure 12 shows an example of a cross-section of a bundle in fabric B. In some areas the fibres are agglomerated and in other less densely packed. For the longitudinal flow, it is a composition of wide and narrow channels connected in parallel. Most of the flow goes through the wide channels increasing the permeability (Fig. 13a). For the transverse flow, the fibre bed acts like different paths in series (Fig. 13b). The narrowest path limits the flow conductivity.

In another study [28], the method of Bechtold [26] was applied to determine the transverse permeability of the 68 tex roving at $v_{f}$ of $58 \%$. The progression of the

Fig. 13 Flow between the fibres

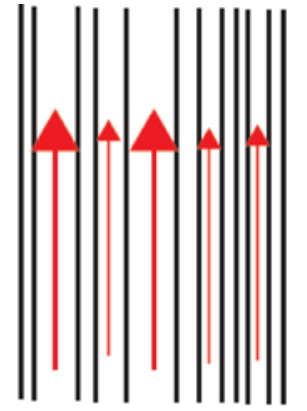

a Longitudinal flow

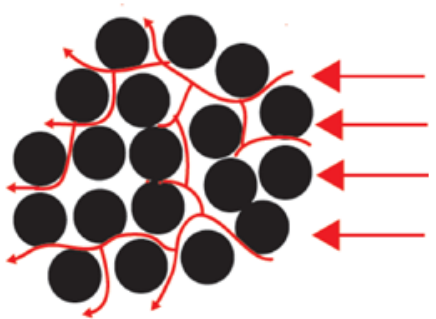

b Transversal flow 
flow front through a distributed fibre arrangement was evaluated with Darcy's law. The fibre arrangement was determined with the Morishita index from cross-sections of composite parts samples. This unsaturated permeability has a value of around $1 \mathrm{E}-14 \mathrm{~m}^{2}$. The permeability varies depending on the gaps width. The corresponding saturated permeability is $6.9 \mathrm{E}-13 \mathrm{~m}^{2}$. This is a good agreement, as the measured saturated permeability is higher than the unsaturated one [29, 30].

\section{Conclusions}

Fibre bundle permeability was measured transversal and longitudinal. The measured values show a high variability and are higher than the calculated ones. This can be explained with discrepancy of the fibres arrangement in theory and reality. In a realistic fibre bed, like in the experiment, some wider and smaller channels between the fibres occur. The wider channels increase the permeability. As the occurrence of these channels is stochastic, the resulting permeability has a certain variability. A realistic prediction of the fibre bundle permeability has to include this stochastic fibre arrangement variations which are very complex. Only the methods of Bechtold [26] and Um [27] seems to be adequate, but these methods require image analysis and experimental work, too. The disadvantage of these methods is, that they give one value for the permeability without any indication of the accuracy like a confidence interval. The confidence interval is very useful for example for the calculation of the impregnation time based on the permeability. The longest possible impregnation time can be given with the confidence interval. The experimental determination gives realistic results with the stochastic correctness if the homogeneous alignment and tortuosity of the rovings are taken into account.

The presented measurement setup enables the experimental determination of the transversal and longitudinal permeability for different fibre volume fractions. The handling of the fibres, winding and laying aligned in the mould, is very conserving on the fibre arrangement. Tortuosity of the fibres is avoided. Thus the fibre arrangement represents the real arrangement in the bundles in a fabric. The comparison of the roving with different thread sizes shows that measurements with roving of a lower thread size have a smaller dispersion.

Acknowledgements The authors gratefully acknowledge support by the Swiss National Science Foundation under contract No. 200020-109109/1.

\section{References}

1. Babu, B.Z., Pillai, K.M.: Experimental investigation of the effect of fiber-mat architecture on the unsaturated flow in liquid composite molding. J. Compos. Mater. 38(1), 57-79 (2004)

2. Slade, J., Pillai, K.M., Advani, S.G.: Investigtion of unsaturated flow in woven, braided and stitched fiber mats during mold-filling in resin transfer molding. Polym. Compos. 22(4), 491-505 (2001)

3. Kang, T.M.K., Lee, W.I., Hahn, H.T.: Formation of microvoids during resin-transfer molding process. Compos. Sci. Technol. 60, 2427-2434 (2000)

4. Schell, J.S.U., Deleglise, M., Binetruy, C., Krawczak, P., Ermanni, P.: Numerical prediction and experimental characterisation of meso-scale-voids in liquid composites moulding, (Submitted to Composites Part A) 
5. Han, K.K., Lee, C.W., Rice, B.P.: Measurement of the permeability of fiber preforms and applications. Compos. Sci. Technol. 60, 2435-2441 (2000)

6. Verheus, A.S., Peeters, J.H.A.: The role of reinforcement permeability in resin transfer moulding. Compos. Manuf. 4(1), 33-38 (1993)

7. Luthy, T., Landert, M., Ermanni, P.: 1d-permeability measurements based on ultrasound and linear direct current resistance monitoring techniques. J. Mater. Process. Manuf. Sci. 10, 25-43 (2001)

8. Williams, J.G., Morris, C.E.M., Ennis, B.C.: Liquid flow through aligned fiber beds. Polym. Eng. Sci. 14(6), 413-419 (1974)

9. Deng, J.L., Zhu, Y.D., H.W.J., Meng, Z.H.: Numerical simulation of micro-infiltration in a fibre bunndle. Key Eng. Mater. 249, 347-350 (2003)

10. Gutowski, T., Cai, Z., Bauer, S., Boucher, D., Kingrey, J., Wineman, S.: Consolidation experiments for laminate composites. J. Compos. Mater. 21, 650-669 (1987)

11. Ranganathan, S.: A generalized model for the transverse fluid permeability in unidirectional fibrous media. Polym. Compos. 17(2), 222-230 (1996)

12. de Wiest, R.J.M.: Flow Through Porous Media. Academic Press, New York (1969)

13. Scheidegger, A.E.: The Physics of Flow Through Porous Media. University of Toronto Press, Toronto (1974)

14. Bear, J.: Dynamics of Fluids in Porous Media. Dover, New York (1988)

15. Dullien, F.A.L.: Porous Media: Fluid Transport and Pore Structure, no. 3. Academic Press, San Diego (1992)

16. Darcy, H.: Les fontaines publiques de la ville de Dijon. Victor Dalmont, Paris (1856)

17. Kozeny, J.: Über kapillare Leitung des Wassers im Boden - Aufstieg, Versickerung und Anwendung auf die Bewässerung. Sitzungsberichte der Akademie der Wissenschaften, Wien136 (1927)

18. Carman, P.C.: Fluid flow through granular beds. Trans. Inst. Chem. Eng. 15, (1937)

19. Cai, Z.: Estimation of the permeability of fibrous preforms for resin transfer moulding processes. Compos. Manuf. 3(4), 251-257 (1992)

20. van den Brekel, L.D.V., de Jong, E.J.: Hydrodynamics in packed textile beds. Tex. Res. J. 59(8), 433-440 (1989)

21. Skartsis, L., Kardos, J., Khomami, L.: Resin flow through fiber beds during composite manufacturing processes. Part 1: Review of newtonian flow through fiber beds. Polym. Eng. Sci. 32(4), 221-230 (1992)

22. Skartsis, L., Kardos, L., Khomami, J.: Resin flow through fiber beds during composite manufacturing processes. Part 2: Numerical and experimental studies of newtonian flow through ideal and actual fiber beds. Polym. Eng. Sci. 32(4), 231-239 (1992)

23. Astrom, B.T., Pipes, R.B., Advani, S.G.: On flow through aligned fiber beds and its application to composites processing. J. Compos. Mater. 26(9), 1351-1373 (1992)

24. Gebart, B.R.: Permeability of unidirectional reinforcements for RTM. J. Compos. Mater. 26(8), 1100-1133 (1992)

25. Lundstrom, S.T., Gebart, B.R.: Effect of perturbation of fibre architecture on permeability inside fibre tows. J. Compos. Mater. 29(4), 424-443 (1995)

26. Bechtold, G., Ye, L.: Influence of fibre distribution on the transverse flow permeability in fibre bundles. Compos. Sci. Technol. 63, 2069-2079 (2003)

27. Um, M.K., Lee, W.I.: A study on permeability of unidirectional fiber beds. J. Reinf. Plast. Compos. 16(17), 1575-1590 (1997)

28. Hurschler, P.: Bestimmung der repräsentativen Faseranordnung im Faserbündel zur Simulation des Mikroflusses Semesterarbeit, ETH Zurich (2004)

29. Shojaei, A., Trochu, F., Ghaffarian, S.R., Karimian, S.M.H., Lessard, L.: An experimental study of saturated and unsaturated permeabilities in resin transfer molding based on unidirectional flow measurements. J. Reinf. Plast. Compos. 23(14), 1515-1536 (2004)

30. Bréard, J., Henzel, Y., Trochu, F., Gauvin, R.: Analysis of dynamic flows through porous media. Part 1: Comparison between saturated and unsaturated flows in fibrous reinforcements. Polym. Compos. 24(3), 391-408 (2003) 